\title{
TTR
}

Traduction, terminologie, re?daction

\section{La traduction mélancolique (sur Paul Celan)}

\section{Alexis Nouss}

Volume 11, numéro 2, 2e semestre 1998

Psychanalyse et traduction : voies de traverse

Psychoanalysis and Translation: Passages Between and Beyond

URI : https://id.erudit.org/iderudit/037341ar

DOI : https://doi.org/10.7202/037341ar

Aller au sommaire du numéro

\section{Éditeur(s)}

Association canadienne de traductologie

ISSN

0835-8443 (imprimé)

1708-2188 (numérique)

Découvrir la revue

Citer cet article

Nouss, A. (1998). La traduction mélancolique (sur Paul Celan). TTR, 11(2),

199-231. https://doi.org/10.7202/037341ar

\section{Résumé de l'article}

La traduction mélancolique (sur Paul Celan) — Dans l'oeuvre de Paul Celan, poèmes et traductions (en majorité des poèmes, du XVIe au XXe siècles, à partir de sept langues) participent d'une même poétique dont la fonction est de retrouver une langue atteinte par la ténèbre du nazisme et d'opérer un travail de deuil. La stratégie scripturaire des traductions, cependant, est spécifique en ce qu'elle permet un dire affrontant le trauma. À l'aide de notions freudiennes, notamment le transfert et la mélancolie, nous étudions ce processus à travers la traduction du commentaire de Jean Cayrol écrit pour le film Nuit et brouillard.
Cedocument est protégé par la loi sur le droit d'auteur. L'utilisation des services d'Érudit (y compris la reproduction) est assujettie à sa politique d'utilisation que vous pouvez consulter en ligne.

https://apropos.erudit.org/fr/usagers/politique-dutilisation/ 


\title{
La traduction mélancolique (sur Paul Celan)
}

\author{
Alexis Nouss
}

Le Colloque Paul Celan aura lieu dans sa ville natale. Sur l'invitation officielle, reçue de l'Institut français d'Ukraine : Tchernovitsi (les Ukrainiens écrivent : Chernivtsy ou Tscherniwzi). $A$ la gare de Bucarest, ma première étape, l'employée me vend un billet pour Cernauti (prononcer : Tsernautsi). Mais dans le train, je rêve ma destination la habsbourgeoise Czernowitz Tchernovitsi-Cernauti-Czernowitz. Ou dans l'ordre historique : Czernowitz-Cernauti-Tchemovitsi, selon les langues des souverainetés successives. Trois villes ou une ville à trois noms? Palimpseste urbain comme celui que dessine Freud au début du Malaise. Pas tant métaphorique pour lui, citoyen de ces terres cacaniennes où les villes, de même que leurs habitants, changeaient souvent de noms. Il le savait bien : " Je suis né à Freiberg, en Moravie, une petite ville de la Tchécoslovaquie actuelle... »

Près de la frontière roumano-ukrainienne, vastes plaines décolorées, soleil pâle, le train s'immobilise pendant deux heures trente. Le long des rails, d'interminables files d'essieux et d'étranges pattes d'araignées montées sur roues. Des paysannes, blondeur des jeunes, les plus âgées le fichu sur la tête, proposent par les fenêtres des œufs et des bouteilles de bière. Un à un, les wagons - les voyageurs à l'intérieur, interdit de descendre et d'aller aux toilettes - sont hissés par les grues-pattes d'araignées et les 
essieux sont changés. On m'explique, en roumain-allemand-anglaisukrainien et par gestes. Les rails des ex-républiques soviétiques n'ont pas la même largeur que les autres pays d'Europe. Mesure de précaution adoptée à la dernière guerre pour prévenir une invasion ferroviaire des armées hitlériennes. Depuis, tout train entrant dans ces territoires ou les quittant est soumis à ce rituel. De l'usage politique de la technologie, quoi de neuf? Mais je me demande : si j'en fais un usage métaphorique, le rituel représente-t-il une traduction, ou son contraire?

L'histoire ne s'absente jamais. Les lieux ignorent le silence des hommes. Demeurent les demeures, lorsque meurent les rumeurs. $\grave{A}$ Czernowitz, deux adresses de Celan. Il résida à la première jusqu'à quinze ans, en 1935. Il habita à la seconde jusqu'en 1942.

Des arbres devant la première maison, 5 rue Wassilkostrasse (aujourd'hui Saksagansky). Sur la facade rose et beige, trop chargée, style tournant du siècle, trois plaques plutôt kitsch en forme de livres, pour ne pas s'y tromper : le lieu d'un écrivain. La seconde adresse, 10 rue Masarykgasse : un petit immeuble gris, austère modernité architecturale des années vingt, similaire à tous les autres de cette rue sans arbres. Pas de flaque, rien. Le décor est nu, impersonnel, c'est-âd-dire sans personne. De là les parents de Celan furent déportés le 27 juin 1942. Quelle plaque, quels mots pourraient dire ce déplacement?

Pourtant c'est la scène primordiale. Justement elle est la scène du vide, de la disparition. Le lendemain matin quand Paul Antschel revient après s'être caché durant la nuit, il trouve des scellés sur la porte. Ce jourlà, il devient Paul Celan.

(Notes d'un joumal de voyage, juin 1998)

La scẻne est vide. Elle met en scène le vide. L'œuvre de Celan le mettra en mots. Des mots pour cette scène vide. Le poème celanien dont les vers tendent de plus en plus à la concision - notamment par la fragmentation des propositions et des mots sur plusieurs lignes - , dont le texte tend de 
plus en plus à la brièveté, fait de la page, en l'occupant minimalement, cette scène vide.

Celan la représente par les deux volets de son écriture : la poésie, assurant la fonction d'une anamnèse', et la traduction, activité qu'il pratiqua tout au long de sa vie, dès l'adolescence. Mais après la guerre et la Shoah, la traduction acquiert une fonction spécifique comme mode scripturaire, débordant le seul transfert inter-langagier pour s'attacher pareillement à l'écriture des poèmes. Dans la logique du terme allemand Obersetzung, la traduction est ce qui fait passer au-delà, de l'autre côté de l'histoire et de la langue, les deux marquées par un trauma condamnant au silence de la mélancolie.

La langue peut devenir étrangère à celui qui la parle et qui n'a pourtant rien d'autre en partage. Celan témoigne d'une double césure, celui de l'individu par rapport à sa langue et celui de lindividu par rapport à son co-locuteur, double blessure infligée par l'histoire : « Elle, la langue, fut sauvegardée, oui, malgré tout. Mais elle dut alors traverser son propre manque de réponses, dut traverser un mutisme effroyable, traverser les

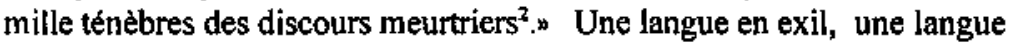
perdue, comme une mère, comme une patrie. On ne peut plus faire confiance au langage quand celui-ci a permis la parole des bourreaux. D'où ce qui passe pour l'obscurité de sa poésie mais qui n'est que l'opacité du langage aujourd'hui, tel qu'il est devenu depuis le passage par le gouffre. D'où l'appel au traduire comme mode fondamental du langage. Faire passer au-delà puisqu'aussi bien la langue allemande, l'originelle, la non-corrompue, la maternelle, est restée de l'autre côté, du côté de l'avant. Le deuil de la langue oblige à trouver une langue du deuil, qui va rendre possible ce travail.

[...] je pense que c'est depuis toujours une espérance du poème, de parler, avec ce langage justement, comme si c'était d'ailleurs - non, je

' Voir Alexis Nouss, "Mémoire et survie : une lecture de Paul Celan ", Études françaises, 34, 1, printemps 1998, et « Das Haus des Vergessens : la poésie comme anamnèse n, Actes du Colloque Celan de Czernowitz 1998, à paraître.

2 " Discours de Brême w, dans Poèmes (tr. John E. Jackson), Le Muy, Éd. Unes, 1987, p.16. 
ne peux plus utiliser ce mot desormais, - comme si c'était au nom d'un autre - qui sait, peut-être au nom d'un tout autre. [...] Le poème est au plus fort quand il est au bord de lui-même; c'est de là qu'il appelle, mais il ne peut plus s'y tenir qu'en s'arrachant sans cesse de son déjà-plus vers son encore 3 .

* De son déjà-plus vers son encore " n'est-ce pas là, dans ce qu'écrit Celan de la poésie aujourd'hui, esquisser une phénoménologie du traduire qui s'articulerait à ce que la psychanalyse nous dit de l'Autre? Si l'historicité de l'écriture celanienne - une langue d'après le langage éclaire le processus traductif, c'est qu'une telle poétique trouve dans la traduction sa dynamique même. Dans la langue perdue et retrouvée mais conservant la trace de sa perte se dessine l'être du traduire, esquissant en retour la tension ontologique désormais attachée au langage.

Il est cependant un autre đeuil dont l'exigence s'avère impossible car lui manque l'objet : la déportation de juin 1942. Il s'agit alors de faire le deuil du deuil, selon une analyse souvent entreprise dans la critique du corpus litteraire de la Shoah ou dans les approches psychanalytiques portant sur les enfants de déportés ${ }^{4}$. L'articulation freudienne entre deuil et mélancolie est ici précieuse, d'où je retiendrai le propos suivant : * Le complexe mélancolique se comporte comme une blessure ouverte attirant de toutes parts vers lui des énergies d'investissement [...] et vidant le moi jusqu'à l'appauvrir complètement $[\ldots]^{5}$ m. La métaphore me retint de son assonnance avec un poème de Celan :

DEIN VOM WACHEN stößiger Traum./Mit der zwölfmal schrauben/förmig in sein/Horn gekerbten/Wortspur.//Der letzte Stoß, den er fuht.//Die in der senk-/rechten, schmalen/Tagschlucht nach oben/stakende Fähre ://sie setzt/ Wundgelesenes über.

${ }^{3}$ * Le Méridien * (tr. Jean Launay), Po\&sie, $\mathrm{n}^{\circ}$ 9, 1979.

'Sur le lien entre la mélancolie et la barbarie du siècle, voir, entre autres, les dernières pages de Jacques Hassoun, La cruauté mélancolique, Paris, Aubier, 1995, et Julia Kristeva, Soleil noir. Dépression et mélancolie, Paris, Gallimard, 1987 (ch. VIII).

$3_{\alpha}$ Deuil et mélancolie , dans Métapsychologie (tr. J. Laplanche et J.-B. Pontalis), Paris, Folio/Essais, 1986, p. 162. 
TON RÊVE encornant à force de veiller./Avec son tracé de mots/créné dans sa corne/filetée douze fois/comme une vis./Le dernier coup qu'il donne.//Dans la gorge du jour/verticale et étroite,/la traille gaffant/vers le haut ://elie fait passer/ce qui a été lu jusqu'à en blesser ${ }^{6}$.

On nous a mis en garde ${ }^{7}$ : übersetzen, traduire, est un verbe inséparable et on ne peut le lire dans le " setzt... über * des deux demiers vers. Pourtant, le syntagme $\alpha[. .$.$] qui ne saurait en aucun cas se traduire$ par * traduit ", passe aussi par-dessus cette impossibilité grammaticale pour faire signe vers la traduction de cette lecture-blessure, passant la frontière vers l'autre côté, du côté de l'autre ${ }^{\mathrm{R}}$. Faire signe : la stratégie même de l'inconscient, fonctionnant uniquement dans ce geste ${ }^{9}$, sans jamais arrêter sa dynamique dans la production de signes, dans une quelconque signification. Tout le poème semble, par son lexique et ce qu'il décrit, évoquer des mécanismes psychiques - le rêve, le tracé de mots, le mouvement vers la clarté et vers le haut - qui se figureraient dans la traduction comme passage de l'autre côté. Elle serait ce qui permet de jouer l'appauvrissement mélancolique du moi dont parle Freud, puisque le traducteur s'efface dans un premier temps devant l'auteur de l'original - la lecture blessante du poème précité -, tout en préservant sa subjectivité, puisque le traducteur produit un texte qui récupérera ses affects. La mélancolie étant liée à la pulsion de mort - provoquée par une mise à mal du narcissime et une " hémorragie libidinale " allant jusqu'à l'acceptation d'une perte du moi -, la traduction ne serait pas tant la mort de l'original, au sens de son remplacement, sa substitution, que la mort

'Traduction de Elfie Poulain, dans Hans-Georg Gadamer, Qui suis-je et qui estu? Commentaire de Cristaux de souffle de Paul Celan, Arles, Actes Sud, 1987, p. 80. Voir aussi la traduction de Michel Deguy et Jean Launay dans Po\&sie, $n^{\circ} 9,1979$, p. 42 . La blessure est un motif récurrent dans les poèmes de Celan.

7Voir la derniêre référence de la note précédente.

Jacques Derrida, Schibboleth. Pour Paul Celan, Paris, Galilee, 1986, p. 98.

${ }^{9}$ Je prends l'expression * faire signe " dans son acception corporelle, m'en autorisant de Celan qui faisait du poème une poignée de mains ( Lettre à Hans Bender *, dans Martine Broda, Dans la main de personne. Essai sur Paul Celan, Paris, Cerf, 1986, p. 109). Quand je fais un signe de la main à quelqu'un, le sens n'est que de ce geste. 
fantasmée du traductant. Pour l'écrivain ${ }^{10}$, la traduction serait la mise en acte de ce fantasme et dans le cas de Celan, le fantasme de sa mort dans les camps, construction psychique faisant de sa vie une survie s'énonçant dans l'écriture. La traduction étudiée plus bas l'illustre exemplairement.

On nous a aussi mis en garde contre toute lecture freudienne de Celan. Sous divers arguments dont le fait que Celan se soit toumé vers la psychiatrie pour apaiser ses maux. Mais Celan lut Freud. Et le cita dans ses poèmes à plusieurs reprises, dont celle-ci où il le fait explicitement, frontalement, dirai-je :

FRANKFURT, SEPTEMBER/Blinde, licht-/bartige Stellwand/Ein Maikäfertraum/leuchtet sie aus.//Dahinter, klagegerastert,/lut sich Freuds Stirn auf,/die draußen/hartgeschwiegene Trine/schießt an mit dem Satz :/ ZZum letzten-/mal Psycho-/logie. W/Die Simili/Dohle/frühstückt.//Der KehlkopfverchluBlaut/singt.

FRANCFORT, SEPTEMBRE.//Cloison mobile à barbe/lumineuse, aveugle./Un têve de hannetons/lillumine.//Derrière elle, tramé de lamentations,/s'ouvre le front de Freud,/une larme/durement tue dehors/jaillit avec la phrase :/a Pour la dernière/ fois de la psycho/logie, $w /$ Le simili-/choucas/déjeune.//L'occlusion laryngale/chante ${ }^{13}$.

Le poème figure dans le recueil Fadensonnen qui prend son titre du poème éponyme d'Atemwende se concluant par : " [...] il y a/encore des chants à chanter au-delà/des hommes ${ }^{\text {i2 }}$ o où se proclameraient un destin du texte traduit, sa survie, de l'autre côté de la mort ou du silence, mais aussi un destin des traducteurs. "L'occlusion laryngale/chante. "Une parole se tait, la parole s'éteint mais reste le texte. Devant la précision pathologique monte un fantasme biographisant qui unirait ces traducteurs atteints, sous diverses formes, d'aphasie : Baudelaire, Mallarmé, Rosenzweig, Larbaud. Sy joindrait Freud, consignant dans L'Interprétation des rêves le a rêve de hannetons " du poème, peut-être

${ }^{10}$ Dont Blanchot a su montrer le rapport étroit à la mort.

"Tr. Bertrand Badiou, dans Po\&sie, 1994.

${ }^{12}$ Choix de poèmes (tr. J.-P. Lefebvre), Paris, Poésie/Gallimard, 1998, p. 235. Voir n. 36 . 
annoncé par un * chant du hanneton * mentionné dans un poème antérieur qui clôt La rose de personne.

Et Kafka, qui consigna dans ses carnets le " Pour la dernière fois de la psychologie! " cité dans le poème et écrivait, l'infection d'origine tuberculeuse ayant gagné le larynx, son dernier texte, "Joséphine la cantatrice ou Le peuple des souris ». À l'heure du silence et de la mort, le chant. Tragique en tant qu'échappant à la raison (" psycho/logie w), inexplicable et irrepressible. Celan oppose-t-il Freud à Kafka? Il les rapproche plutôt, déscientifisant le freudisme et retenant de celui-ci le jeu poétique. Le hanneton se dit en allemand " scarabée de mai w, Maikäfer, Käfer où s'entend la syllabe initiale de Kafka et qui désigne un insecte - le cafard français n'est pas loin - qui pourrait bien être celui sous l'apparence duquel "Un matin, au sortir d'un rêve agité, Grégoire Samsa s'éveilla... "(La métamorphose, tr. Alexandre Vialatte).

Freud et Kafka mais aussi Celan ${ }^{13}$, unis dans un même destin liant histoire et écriture : Francfort, la ville accueillant leurs éditeurs respectifs, et Francfort, ville allemande, en septembre, premier mois de f'automne qui pour Celan symbolise la saison où l'histoire s'enténébra.

Cette apparition de la figure de Freud dans la poésie celanienne m'incite, pour ce qui est de la traduction, à deviner ou à chercher derrière le traducteur ce que je nommerais un traductant ${ }^{14}$. Celui-ci serait l'agent et l'objet d'un travail de la traduction's, ensemble de stratégies perlaboratrices semblables à celles que mettent en œuvre le rêve, le deuil, la mélancolie. Ou le texte. Perlaboration : Durcharbeitung, travail à

\footnotetext{
${ }^{13}$ Le choucas du podme se dit en tchèque kavka et Cetan remarqua que le nom hébreu de Kafka, Amschel, est proche de son propre patronyme, Antschel. Jeu identitaire entre les simili-noms. Voir la conclusion du présent article. Quant à l'occlusion laryngale, n'entre-t-elle pas aussi dans la pathologie d'un suicide par noyade?
}

${ }^{14}$ Antoine Berman avait déjà posé dans son analyse de la traduction une « pulsion du traduire * dont il revendiquait l'inspiration freudienne de la formulation (L'Épreuve de l'étranger, Paris, Gallimard, 1984, pp. 21 ss. et pp. 283 ss. ).

${ }^{15}$ Dans la logique d'un tel travail, traductant pourrait aussi se dire tradactant. 
travers, préposition durch traduisible par le trans de translatio ${ }^{16}$. Au demeurant, le travail du deuil, du rêve, etc., n'accomplit-il pas une traduction ${ }^{17}$ ? Si bien que, de même que Jakobson affirme que la traduction interlinguistique est la traduction proprement dite, le travail traductif serait le travail proprement dit, le travail du travail, celui qui dit le propre, c'est-à-dire à la fois l'essentiel et le singulier. Il n'est dès lors pas étonnant que Celan se révèle le plus dans ses traductions, et notamment celle que j'étudierai plus bas.

À la question "Pourquoi traduire? " qui se pose pour nombre d'écrivains-traducteurs, qui se pose pour Celan devant l'ampleur de son cuvre traductive, le phénomène d'identification est une réponse immédiate - Baudelaire et Poe, Celan et Mandelstam ${ }^{1 B}-$ qui doit se prolonger, et s'expliquer, par la considération de ce que j'appellerai un transfert traductif grâce auquel, sous la protection d'un tel lien privilégié, le sujet traductant met au jour des signifiants fantasmés qu'il " reconnaît " dans l'original ${ }^{19}$. La notion est quasi tautologique puisqu'une des traductions de l'allemand Übertragung, transfert, n'est autre que traduction, le long de la chaîne sémantique transmission, transport, transcription, etc. On ne traduit bien que ce (ceux) qu'on aime, assène la pédagogie de la traduction. De même que l'analysant est, d'emblée, prêt au transfert, le traducteur est prêt à trouver dans le texte de départ de quoi nourrir son investissement en même temps qu'il l'investit de ses affects. George Steiner exprime une idée similaire en utilisant un autre lexique, psychologico-religieux :

[Le parcours herméneutique ou traductif] s'ouvre sur un élan de confiance, où tout se déclenche, une profession de foi, soutenue par

${ }^{16}$ Celan fait un usage répétitif de la préposition (hindurchgehen-traverser) dans son * Discours de Brême *. Voir supra, la citation de la n. 2.

${ }^{17}$ Voir, entre autres, Didier Anzieu, Le corps de l'cuvre, Paris, Gallimard, 1981.

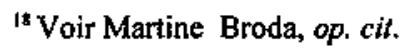

${ }^{19}$ Une telle motivation soutient une autre forme de transfert à l'œuvre dans les poèmes de Celan : la citation, exacte ou non, d'autres auteurs. Par exemple , Vertaine dans a Huhediblu $\bullet$ de La rose de personne. 
l'expérience antérieure mais fragile au niveau épistémologique et risquée sur le plan psychologique, dans la densité signifiante, le x sérieux $\approx$ du texte qui vous fait face ou plutôt vous tient tête. On risque un bond en avant : on concède d'emblée qu'il y a $\alpha$ là-dedans quelque chose à comprendre, que le passage ne se fera pas à vide ${ }^{20}$.

Positif ou négatif, selon la distinction freudienne, le transfert traductif l'est aussi. D'une part, la traduction va produire des énoncés absents ailleurs (par exemple, pour Celan, dans ses poèmes), dût-elle les inventer dans l'original, et d'autre part, elle va omettre ou transformer certains énoncés, témoignant d'une résistance révélant des interdits. Les deux versants du transfert traductif seront abordés dans l'analyse qui suit. L'ambition ou la tentation, souvent constatée, des traducteurs de tout traduire d'un auteur témoignerait de la non-résolution du transfert, en un phénomène similaire à celui de la cure.

La faute de traduction se comprendrait similairement ${ }^{22}$. Lorsque le traducteur commet une faute, le traductant s'y commet. Il voit autre chose dans le texte que son énoncé manifeste. Mais cette méprise qui le déprend du sens obligé n'est pas stérile. Elle lui fait trouver dans la langue autre ce qu'il cherchait ou cachait dans la sienne. L'übersetzen comme forme d'übersehen ${ }^{22}$.

Le lien qu'établit le freudisme entre le transfert et la répétition, le premier comme mode ou effet de la seconde, suggère de définir la

${ }^{211}$ Après Babel. Une poétique du dire et de la traduction (tr. Lucienne Lotringer), Paris, Albin Michel, 1978, p. 277. Dans Passions impunies (tr. Pierre-Emmanuel Dauzat et Louis Evvrard), Paris, Gallimard, 1997, il parle de " pari cartésien " (p. 170) et de * saut a priori * (p. 195). Dans Errata. Récit d'une pensée (tr. P.-E. Dauzat, Gallimard, 1998), il parle de * croyance opératoire * (p. 139). Ce pari sur le sens est la thèse fondamentale qu'il développe longuement dans Réelles présences. Les arts du sens (tr. Michel R. de Pauw), Gallimard, 1991.

${ }^{21}$ Sur la faute de traduction comme mécanisme inconscient, voir François Peraldi, Meta,« Psychanalyse et traduction $\nsim, 27:$ 1, 1982, p. 23.

${ }^{22}$ Sur cette notion, voir Jean-Michel Rey, Des mots à l'auvre, Paris, AubierMontaigne, 1979. Sur le signifiant trouvé dans la parole de l'autre, voir René Kaès, La parole et le lien, Paris, Dunod, 1994. 
traduction non plus tant en termes de reproduction mais de répétition, ce qui brouillera la stérile opposition répétée à satiété - répétition qui, à son tour, devrait être interrogée - dans la théorie traductologique entre l'esprit et la lettre, la fidélité et la liberté. Les deux approches seront désormais deux stratégies différentes empruntées par la dynamique de répétition : le litterralisme correspondant à un tranfert positif, la traduction * libre »à un transfert négatif, les deux indiquant le degré de résistance manifesté par le sujet traductant dans cette tentative de résolution (acceptation ou effacement) du trauma en quoi consiste la répétition.

Un autre poème du recueil Fadensonnen cite exactement le lexique freudien sur ces points en offrant comme une narration poétique du fonctionnement inconscient :

...AUCH KEINERLEI//Friede.//Graunächte, vorbewußtkühl./Reizmengen, otterhaft,/aufBewuftseinsschotter/unterwegs zu/Erinnerungsbläschen.//Grau-in-Grau der Substanz.//Ein Halbschmerz, ein zweiter, ohne/Dauerspur, Eine Halblust./Bewegtes, Besetzes./Wiederholungszwangs-/Camaïeu.

... ET AUCUNE SORTE/de paix.//Grises-nuits, préconsciemmentfraîches./ Des quantités de stimulation, comme des otaries,/sur le gravier de la conscience/en chemin vers/des petites bulles de souvenir.//Gris-en-gris de la substance.//Une moitié de douleur, une seconde, sans/trace durable, à mi-chemin/ici. Une moitié de plaisir./En chemin, en place.//Camaîeu/de contrainte de répétition ${ }^{23}$.

Pour achever sur ce qui ne peut l'être, la répétition, ce poème de Contrainte de lumière :

VORGEWUSST blutet/zweimal hinter dem Vorhang,//Mitgewußt/perlt.

${ }^{23}$ Gesammelte Werke, t. II, Frankfurt am Main, Suhrkamp, 1983, p. 201 (ma trad.). Wiederholungszwang est habituellement traduit par compulsion ou automatisme de répétition. Pour une analyse détaillée des emprunts à Freud, voir GeorgMichael Schulz, "fort aus Kannitverstan". Bemerkungen zum Zitat in der Lyrik Paul Celans ", Text + Kritik, $n^{\text {us }} 53 / 54$, janvier 1977. Pour une analyse d'un exemple de répétition scripturaire, voir John Felstiner, Paul Celan : Poet. Survivor, Jew, New Haven and London, Yale University Press, 1995, pp. 83 ss. 
PRESCIENCE saigne/deux fois derrière le rideau,//connivence/perle $e^{24}$.

Et celui-ci, de Part de neige :

UNLESBARKEIT dieser/Welt. Alles doppelt./Die starken Uhren/geben der Spaltstunde recht,/heiser.//Du, in dein Tiefstes geklemmt,/entsteigst dir/fîr immer.

ILLISIBILITÉ de ce/monde. Tout se redouble.//Les solides horloges/justifient l'heure divisée,/d'un son rauque.// Toi, coincé en ton plus profond/monte hors de toi/pour toujours ${ }^{25}$.

Pour ce qui est de Celan, le rapport qu'établit Freud dans $A u$-delà du principe de plaisir entre la répétition et la pulsion de mort trouve une totale pertinence puisque la traduction lui permet d'en dire les affects, autrement refoulés, alors qu'ils sont essentiels dans son parcours psychobiographique et dans son projet d'écriture. Les poèmes le disent parfois : La demi-mort,/allaitée avec notre vie,/était là tout autour de nous vraie d'images de cendre $-[\ldots]^{26}$.

En outre, la traduction intensifie le mécanisme propre a l'expression artistique de projection en un double puisque traduire est objectiver cette projection. Le gain s'accroît encore dans le cas de Celan du rapport à la mort. "Le "double" qu'est l'art, comme tout double, érigé pour vaincre la mort, se transforme lui-même en image de la mort. Le jeu de l'art est un jeu de la mort, qui implique toujours déjà la mort dans la vie, comme force d'épargne et d'inhibition ${ }^{27}$. " Pour Celan, l'image de la mort renvoie ả une double figuration. Celle de sa propre mort où se joue le syndrome du survivant (au sens large : non le rescapé mais celui qui aurait dû périr de par son identité et qui, en quelque sorte, a échappé ou survécu à sa propre histoire), celle de ses parents, disparus, sans traces de

${ }^{24}$ Contrainte de lumière (tr. Bertrand Badiou et Jean-Claude Rambach), Paris, Berlin, 1989, pp. 44-45.

${ }^{25}$ Gesammelte Werke, t. II, p. 338 (ma trad.).

${ }^{26}$ " À Prague ", dans Choix de poèmes, p. 259.

${ }^{27}$ Sarah Kofman, L'Enfance de l'art, Paris, Payot, «Petite Bibliothèque Payot», 1975 , p. 175. 
la disparition, comme déportés. Le transfert traductif permet d'incorporer ${ }^{28}$ les figures parentales en tant que mortes et de pallier au deuil impossible. De sorte que l'écriture traductive servira à la fois à traduire la perte en la figurant ${ }^{29}$ - rapport de la traduction à l'original - et à la compenser.

DIE SPUR EINES BISSES im Nirgends./Auch sie/mußt du bekämpfen, $/ v o n$ hier aus.

LA TRACE D'UNE MORSURE dans le nulle part./Elle aussi/tu dois la combattre,/à partir d'ici ${ }^{30}$.

Ludiquement, je répéterai : là où il y avait, dans l'espace du nulle part, une perte incertaine, le travail traductif permet de lutter et de retrouver, dans le texte advenu, la trace ... d'une mort sûre.

Sur les cinq tomes des Eirvres complètes de Celan publiées par Suhrkamp, deux en recueillent les traductions, textes poétiques pour la plupart, plus d'une quarantaine d'auteurs, du XVI ${ }^{e}$ au $\mathrm{XX}^{e}$ siècles, à partir de sept langues (russe, anglais, italien, portugais, roumain, hébreu, français) et un volume uniquement consacré à la vingtaine d'auteurs francophones. Divers textes traduits pourraient pertinemment s'offrir à l'analyse (s'imposent les Feuillets d'Hypnos de Char ou Ecce Homo de Michaux, directement liés à l'expérience de la dernière guerre mais aussi d'autres poèmes de Supervielle ou de Dupin marqués par la mort et la perte). Je m'attacherai ici, de manière non exhaustive, à la traduction qu'a faite Celan du texte écrit par Jean Cayrol en commentaire du film d'Alain

${ }^{28}$ Le terme renvoie aux travaux de Maria Torok et Nicolas Abraham. Voir * Introjecter-Incorporer. Deuil ou Mélancolie ", Nouvelle Revue de Psychanalyse, automne 1972.

${ }^{29}$ De même que l'œuvre d'art n'exprime pas le fantasme, majs le constitue dans et par la répétition.

${ }^{30}$ Fadensonnen, Gesammelte Werke, t. II, p. 117, Traduction (modifiée) de J. E. Jackson, op. cit., p. 53. 
Resnais Nuit et brouillard ${ }^{31}$. Unique occurrence où le nom de Celan signe un texte dont le seul objet est la Shoah, alors que par ailleurs toute l'écriture poétique en est motivée sans la mentionner et qu'elle vise à dire limpossibilité de cette mention. La Shoah opère donc comme un trauma, qui va enclencher le processus de répétition, ici la répétition traductive. Exemplaire donc d'un travail de la traduction, visant à une mise en scène et une narrativité répondant aux indications du fantasme, stratégies diverses et parfois contradictoires (répondant à l'ambivalence foncière de lénoncé fantasmatique), révélant les nuances contrastées du refoulement, de l'évitement et de la figuration. Ce que la poésie cèle et recèle, la traduction en décèle le secret. Je ne traiterai cependant pas, faute de place, des similitudes entre cette écriture traductive et la formalisation textuelle des poèmes ${ }^{32}$, ni de la subversion des catégories spatiale et temporelle, propres à la représentation du trauma ${ }^{33}$.

Travail traductif au service d'un sujet meurtri y trouvant la possibilité de dire ce qui a été tu, l'intraduit caché et protégé par l'inconscient. Le texte nous révèle ce dévoilement. "Cette réalité des camps, méprisée par ceux qui la fabriquent, insaisissable pour ceux qui la subissent, c'est bien en vain ${ }^{34}$ qu'à notre tour, nous essayons d'en découvrir les restes " (p. 80). Pas pour le traducteur : "Die Wirklichkeit der Lager : die sie geschaffen haben, ignorieren sie, und die sie erleiden, können sie nicht fassen. Und wir, die wir nun zu sehen versuchen, was übrig bleibt... "[La réalité des camps : ceux qui l'ont produite l'ignorent et ceux qui la subissent ne peuvent la saisir. Et nous qui cherchons maintenant à voir ce qui reste...] (p. 81). Le découragement ou l'impuissance affichés dans l'original sont déniés et effacés dans le texte de Celan. Négation

${ }^{31}$ Gesammelte Werke, t. IV, pp. 76-99.

${ }^{32}$ Pour une esquisse de ce rapprochement, voir mon article $~ *$ Dans la ruine de Babel : poésie et traduction chez Paul Celan », TTR, vol. IX, $\mathrm{n}^{\circ} 1,1996$.

${ }^{33}$ Pour un exemple d'analyse de telles représentations de la Shoah comme trauma, voir Ginette Michaud, * Résistances du récit (Kofman, Blanchot, Derrida) *, Biffures, uL'Étonnement* nº 2,1999 (à paraître).

${ }^{34}$ Le soulignement indique les éléments sur lesquels portent les modifications entre les deux textes. 
d'une négation, d'aucuns la qualifieraient d'erreur de traduction alors qu'elle est porteuse de vérite ${ }^{35}$. " [W]as übrig bleibt ", expression usuelle mais dont l'attestation lexicale ne doit pas masquer ce qu'elle atteste : l'insistance d'un redoublement sémantique, que l'on pourrait traduire : * ce qui reste en reste " ou * ce qui reste de reste ${ }^{36} \%$. Ce reste, intolérable, insaisissable, imprononçable, demeure cependant à portée de langage par le transfert traductif. Au demeurant, toute traduction a pour fonction, une fois le contenu informatif transmis, de dévoiler ce qui reste intraduisible, de traduire par défaut ce qui ne peut l'être, le « noyau de pur langage ${ }^{37}$ " qui fait l'essence de tout texte.

La traduction permet d'affronter l'horreur de l'histoire. Dans les demières lignes du texte ${ }^{33}$, là où Cayrol parle de la persistance du * vieux monstre concentrationnaire " (p. 96), Celan supprime l'adjectif et écrit « die Rassenwahn " (p. 98), la folie raciale ou raciste, utilisant le terme Rasse qu'il n'emploie jamais ailleurs, lui substituant Geschlecht (descendance, génération, sexe) ou Stamm (souche, lignée). Levée d'une censure, retour traductif d'un refoulé.

${ }^{35}$ Voir supra.

${ }^{36}$ N jesessité de rapporter ici cette définition de Derrida dans son livre sur Celan : * ce reste sans reste qu'on appelle cendre * (op. cit., p. 73). Le thème du reste est central chez Celan et fait de la survivance une condition non plus historique mais ontologique. La possibilité de la poésie (après Auschwitz) en est tributaire. Parmi toutes les occurrences citables, celles-ci : * Singbarer Rest ", " Reste chantable *, titre du poème dont la dernière strophe dit : " Lévre privée du pouvoir de parole, fais savoir/qu'il se passe toujours, encore, quelque chose,/non loin de toi * (Choix de poèmes, p.243) et « [...] il y a/encore des chants à chanter au-delàddes hommes * (ibid., p. 235) Le reste celanien s'éclaire d'une philosophie de la trace (Lévinas, Derrida). Y en a-t-il d'autres pour approcher la Shoah?

37 Selon l'expression de Walter Benjamin, * Die Aufgabe des Übersetzers ", * L'abandon du traducteur * (tr. Laurent Lamy et Alexis Nouss), TTR, vol. X, $n^{\circ} 2$, 1997. La logique traductive chez Benjamin participe également de l'après coup. Proximité attendue : tant dans sa pensée de l'histoire que du langage, Benjamin, " né sous le signe de Saturne ", a fait de la mélancolie une catégorie épistếmologique.

${ }^{38}$ L'observation en a été faite par Felstiner, op. cit., p. 93. 
Négation d'une négation, refus du refus, dissiper le brouillard pour affronter la nuit, anamnèse scripturaire qui est l'acceptation douloureuse d'un principe de réalité devant lequel s'élève une double résistance : celle, habituelle, au principe de réalité en général, et celle, spécifique, au mode de manifestation d'une réalité particulière, si particulière qu'elle en bouleverse et le langage (du) conscient ${ }^{39}$ et celui de l'inconscient : l'imaginaire même ne répond plus ${ }^{40}$.

Alors le monde véritable, celui des paysages calmes, celui du temps d'avant, peut bien apparaittre au loin, pas si loin. Pour le déporté, c'était une image. (p. 86)

Worauf dann auch die eigentliche Welt, die der stillen Landschaften, sie der Zeit vorher erscheinen kann - ganz nahe sogar./Für den KZHäfling besitzt sie keine Wirklichkeit. [Alors le monde véritable, celui des paysages caimes, celui du temps d'avant peut apparaître - même tout proche./Pour le déporté (Häfling) ${ }^{41}$ il ne possède aucune réalitéb] (p. 87)

La traduction refuse de parler, même négativement, d'image. Une inversion insistait auparavant sur l'invalidité de la notion même d'image : là où Cayrol, évoquant les blocks des déportés, écrit a [...] aucune description, aucune image ne peuvent leur rendre leur vraie dimension [...] " (p. 82), Celan traduit : " kein Bild, keine Beschreibung * [aucune image, aucune description] (p. 83). La réalité dans sa terrifiante technicité : " gaz zyklon " dit Cayrol (p. 92), Celan précise : «Zyklon B" (p. 93).

${ }^{39}$ Le thèrne de limpuissance du langage à rendre la réalité du camp est récurrent dans la littérature de la Shoah. Voir notamment Primo Levi, Élie Wiesel, Robert Antelme. Le désastre du réel, c'est aussi la défaite et du symbolique et de l'imaginaire.

${ }^{40}$ Comment le ferait-il quand, les deux étant liés, le corps a disparu? Disparition du corps et de son nom dont témoigne la traduction de Celan (voir infra).

${ }^{4}$ Sur cette traduction, voir infra. Le choix du terme répond à la mêtne motivation quant à la réalité. 
Ce qui est en question tient au statut de réalité du camp, tel que le reste du monde s'en trouve vidé $e^{42}$. L'espace outre-concentrationnaire n'a pas plus de réalité représentable que le temps antérieur, mis en italiques par la traduction pour en souligner le caractère hypothétique. Horsfonction, l'imaginaire l'est également à l'intérieur du monde concentrationnaire. Décrivant la construction des camps, Cayrol écrit : " Pas de style imposé. C'est laissé à l'imagination. Style alpin, style garage, style japonais, sans style "(p. 79). Celan écrit, avec omission : "Kein vorgeschriebener Baustil,/ Alpenhüttenstil,/ Garagenstil,/ Pagodenstil,/ohne Stil "[Pas de style de construction imposé,/Style alpin,/Style garage,/Style pagode/sans style] (p. 79). Refus de

\footnotetext{
${ }^{42}$ Levi comme Semprun développent l'idée du camp comme seule réalité, le reste, depuis, étant un rêve, une parenthèse (d'où la signification particulière du suicide chez les écrivains de la Shoah). Mais la problématique est complexe car ces mêmes auteurs trouvent dans l'imaginaire le seul recours expressif. Antelme

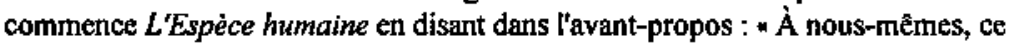
que nous avions à dire commençait alors à nous paraître inimaginable. [...] Nous avions donc bien affaire à l'une de ces réalités qui font dire qu'elles dépassent l'imagination. Il était clair désomais que c'était seulement par le choix, c'est-à-dire encore par l'imagination que nous pouvions essayer d'en dire quelque chose (Paris, Gallimard, * TEL, 1978, p. 9).

Deux controverses récentes viennent alimenter ce débat. La première éclata en l'automne 1998 autour de l'ouvrage Fragments. Une enfance (Paris, CalmanLévy, 1997) de Benjamin Wilkomirski, récit autobiographique de la déportation d'un enfant et de sa remémoration traumatique, salué par la critique et récipendaire de nombreux prix, soupçonné d'être un faux dû à la plume d'un musicien suisse n'ayant pas connu le génocide. Certes, le révisionisme se régalera de ce qu'un écrit sur la Shoah se révèle être une imposture et, politiquement, on ne pourra qu'en condamner la parution. Mais, philosophiquernent, le phénomène génocidaire insaisissable dans les catégories intellectives habituelles, la mort nazie incompréhensible comme terme naturel de la vie, il faut admettre que, à la limite, seule une biographie fictive pouvait rendre compte d'une thanatographie rendue fictive à la fois par l'impossibilité intrinsèque d'en rendre compte et par le soin des nazis à en effacer toutes traces. La seconde polémique toucha la production cinématographique en 1998 avec la sortie de La vie est belle de Roberto Benigni et Train de vie de Radu Mihaleanu. L'humour est-il blasphématoire? Il est bien plutôt, par sa dé-raison, le seul moyen d'approcher l'opacité d'un réel à jamais insymbolisable. Chaplin tournant Le dictateur l'avait déjà compris. Le réalisme hollywoodien, agrémenté de l'inévitable touche optimiste, de La liste de Schindler est autrement scandaleux.
} 
l'imaginaire si la seule mention de celui-ci filtre encore la réalité. S'étonnant de la résistance du corps humain, Cayrol écrit : [ [...] les mains couvertes de pansements travaillent "(p. 86). Omission dans la traduction : * [...] die Hände sind rege * [les mains sont actives] (p. 87). Le mal et la souffrance restent entières, ne connaissent de répit. La blessure saigne. Pas de pansements dans le camp, pas de pansements au camp.

Négation (traductive) d'une négation dans cet autre passage : * "Transports noirs" qui partent à la nuit et dont personne ne saura jamais rien. $"$ (p. 86). Or ce rien a une réalité que la traduction trouve le courage, par substitution à l'original, de nommer : « Die "Dunkeltransporte" -ihr Ziel sind die Krematorien " [Les a transports obscurs " - leur destination est les crématoires] (p. 87). Substitution d'autant plus remarquable, révélant en cela le travail psychique, que l'obscurité, telle la noirceur nocturne, est, dans les poèmes de Celan, un motif récurrent exprimant le mode de manifestation du réel après le désastre, comme la vérité qui, dans le freudisme, se donne dans ses dissimulations.

En même temps, la traduction avoue l'impossibilité du dire : « De ce dortoir de briques, de ces sommeils menacés, nous ne pouvons que vous montrer l'écorce, la couleur « (p. 82); * Von Gefahren umlauerter, backsteinfarbener Schlaf... "[Du sommeil entouré de dangers, à la couleur de briques...] (p. 83). On ne montre rien, on ne peut rien montrer, la traduction ne sert qu'à évoquer ce qui aurait dû être montré, mais l'omission en marque l'impossibilité. Le silence traductif pose la limite de la représentation. L'insoutenable est atténué par une modification traductive :

Un crématoire, cela pouvait prendre à l'occasion un petit air de carte postale. Plus tard - aujourd'hui - des touristes s'y font photographier. (p. 92)

Ein Krematorium : das nimmt sich gelegentlich ganz nett aus./Später - heute - lassen Touristen sich davor photographieren. [Un crématoire : cela prend à l'occasion un air tout à fait coquet. Plus tard - aujourd'hui -- des touristes s'y font photographier.] (p. 93)

Résistance, et pourtant la blessure reste ouverte : le présent se substitue à l'imparfait et le butoir rhétorique de " pouvoir * est supprimé. 
Douleur de ce dire, marquée par la substitution traductive d'un signe. Citant les a préceptes " qui ornaient le camp, Cayrol en souligne l'ironie en ironisant à son tour : " "UN POU C'EST LA MORT". Et un S.S. donc! " (p. 84). Celan ne s'autorise pas telle distance enonciative : " LAUS DEIN TOD". Und ein SS-Mann? " (p. 85). Un point d'interrogation pour un point d'exclamation : minorisation de la parole, la plainte et non plus l'indignation, plainte mélancolique où l'affect identificatoire donne voix au traumatisme. Blessure ouverte. Cayrol décrit les conditions dans les wagons de déportés : [...] ni jour, ni nuit, la faim, la soif, l'asphyxie. Ia folie " (p. 78); * kein Tag, keine Nacht, Hunger, Durst, Wahnsinn. Ersticken "(p. 79). Celan corrige l'ordre des maux en inversant les deux derniers termes et rétablit une succession où l'asphyxie prend la dernière place, celle de la fin, comme elle la prit dans les chambres à gaz, fin de la représentabilité aussi (le paragraphe continue chez Cayrol, la ligne s'arrête là pour Celan). Folie et/puis asphyxie : biographème et thanathème ${ }^{43}$ pour Celan, les crises répétées qui le menaient dans les cliniques et le suicide par noyade. Freud ( $*$ Deuil et mélancolie $*): *[. .$.$] l'énigme de la tendance$ au suicide qui rend la mélancolie si intéressante - et si dangereuse ${ }^{44} n$.

“ On réussit à écrire, à prendre des notes, à exercer sa mémoire avec des rêves. On peut penser à Dieu * (p. 88); « Man bringt es fertig zu schreiben,/mit seinem Gedächtnis zu spielen../Man kannt an Gott denken... "[On réussit à écrire,/à jouer avec sa mémoire.../On peut penser à Dieu...] (p. 89). La traduction sépare les deux énoncés, dénouant ce qu'ils ont en commun, la description distante de ce qu'il demeure encore possible de faire. Et la transfomation du premier est signifiante, où la traduction traduit son travail interne. La fonction informative (prise de notes) est omise, il s'agit bien d'écriture au sens plein, comprise dans ce qu'elle accomplit au niveau psychique : le jeu de la mémoire, lexique proprement freudien. Dans les poèmes, l'ecriture est la traduction du trauma. Ici, la traduction en est l'écriture.

\footnotetext{
43 J'indique par ce terme un motif récurrent dans sa poésie renvoyant à ce qui sera sa mort. Voir mon livre à paraître, Le déplacement. Poésie et traduction chez Paul Celan.
}

${ }^{44}$ Op. cit., p. 160. 
Écriture traductive, c'est-à-dire transformatrice, d'autant plus nécessaire que le titre ouvre la blessure. Aucune échappatoire : traduire le titre "Nuit et brouillard " (pp. 76-77) ne peut être qu'une nontraduction, la seule citation du réel, la fulgurance du trauma : $\propto$ Nacht und Nebel ${ }^{45}$. Le syntagme fut le nom donné par les nazis a l'opération d'extermination. Il perd la vague aura romantique qu'il peut acquérir en français. Il revient alors a la traduction du texte de mettre en œuvre les stratégies fantasmatiques permettant son écriture en allemand après un transfert sur l'original français.

Ce qui est impossible en allemand, ou impossible directement, selon l'usage normal, Le style des poèmes celaniens, fait de dislocations sémantiques et formelles, montre cet impossible. Car la langue allemande est interdite : elle ne peut plus dire ce qu'elle a historiquement dit, ne peut même plus dire cette histoire, invalidée par cette histoire ${ }^{46}$. Interdite au sens de non permise, car elle appartient aux maittres et bourreaux (un décret nazi en défendit d'ailleurs l'usage aux Juifs), mais aussi interdite au sens de : figée, inopérante, paralysée, médusée (comme dans lexpression " en rester interdit ").

Le trauma s'annonce et s'énonce dès le titre, adoptant donc une position fondatrice, mais de complexe façon, dans un chiasme familier du psychisme, puisque le trauma est dit à partir d'une double altérité : le texte

\footnotetext{
${ }^{45}$ Nacht est, après Wort (mot ou parole) et $A u g$ (œil) le substantif le plus fréquent du lexique des poèmes celaniens. Il n'est dans l'œuvre poétique jamais associé à Nebel. Ce dernier terme, en revanche, est lié au langage : "Du sangst auch ein Lied, und wir flochten ein Gitter im Nebel "; * C'est que tu chantais, et nous tissions une grille dans le brouillard * (Pavot et mémoire, tr. Valérie Briet, Paris, Christian Bourgois éditeur, 1987, pp. 32-33; voir Sprachgitter (grille de langage ou de parole), titre du quatrième recueil de Celan). "Sichttunnels, in/den Sprachnebel geblasen i; « des tunnels de visibilité, soufflés/dans le brouillard du langage (Contrainte de lumière, p. 159). Le langage serait ce qui perce le brouillard. Dans de nombreuses occurrences du mot nuit, le langage exerce la même fonction. Mais il faut la traduction du texte de Cayrol pour que la censure soit déjouce et les deux termes réunis.
}

${ }^{46}$ Seul poème explicitement lié à la Shoah, la célèbre " Fugue de mort * (dans Pavot et mémoire, p. 85). Mais on sait combien Celan s'opposait à sa diffusion pour un usage " pédagogique w. 
français de Cayrol renvoyant au discours en allemand du nazisme. Dans le texte celanien, l'événement traumatique frappe l'énoncé de sa marque en allemand et, simultanément, se donne en sa propre répétition puisque le syntagme $\mathrm{Nacht}$ und Nebel est la traduction du titre en français. Clivage originaire d'une orthodoxie psychique imparable : à la fois dédoublement avec projection (Celan traduisant Cayrol) et répétition (la traduction). $\mathrm{Si}$ efficace que Celan, dans le corps du texte, opérera un choix syntaxique lui permettant de ne pas dissocier le syntagme : " Un second [choix] est fait à l'arrivée dans la nuit et le brouillard * (p. 78); * Eine zweite folgt am Bestimmungsort ${ }^{47}$, bei Nacht und Nebel " (p. 79). La préposition bei n'appelant pas d'article défini, le syntagme reste intact dans la force connotative de son affect.

Là où la poésie de Celan ne dit pas la Shoah, la traduction le fait et, plus encore, la traduction se fait poésie. D'emblée, pour le regard et la diction, la typographie présente le formalisme associé au texte poétique : non plus la suite de paragraphes de prose de Cayrol, de longueur variable ${ }^{48}$, mais des phrases et des propositions, des segments de phrases et de propositions, disposées sur des lignes distinctes comme des vers libres, regroupés en strophes, reformant parfois des paragraphes, ne reproduisant pas les sections de l'original pour adopter d'autres césures. Comme des vers libres, ces paroles disent l'enfermement infini. Le texte en prose glisse ainsi vers un formalisme qui, l'apparentant au discours poétique, permet plus aisément, au niveau des signifiants graphiques et sonores, le travail d'association et de déplacement propre à l'élaboration fantasmatique. Mais ce travail est préalablement rendu possible par la levée d'inhibition liée au transfert traductif.

${ }^{47}$ Gain traductif : Bestimmung signifie destination, mais aussi décision, arrêt, prescription. La traduction permet de dire que la destination était destin, non pas un lieu de mort, mais la mort comme lieu.

${ }^{48}$ Le texte a été seulement publié pour la première fois en 1997 (Paris, Fayard, collection * Libres *) alors que le film est sorti en 1956. La version allemande de Celan paraît déjà en 1970 à Zürich, avant donc le texte français. Celui-ci, cependant, est reproduit en 1983 dans le volume des CErvres complètes regroupant ses traductions à partir du français, paru en Allemagne. Destin éditorial qui en dit long sur les refoulements et les censures. 
Dès les premières lignes, la suppression d'une marque adverbiale exprime un aspect majeur de ce travail, l'acceptation d'un principe de réalité autrement nié :

Même un paysage tranquille, mêrne une prairie [...], même un village pour vacances, avec une foire et un clocher, peuvent conduire tout simplement à un camp de concentration. (p. 76)

Auch ruhiges Land,/Auch ein Feld [...],/Auch ein kleiner Ferienort mit Jahrmarkt und Kirchturm/kann zu einem Konzentrationlager hinfuhren [peut conduire à un camp de concentration]. (p. 77)

Ce qui pourrait passer pour un jugement subjectif, l'opinion d'un auteur, est supprimé : s'établit un constat, s'énonce le fait objectif, historique. Mais une histoire étrangement amputée : trois dates dans le texte de Cayrol : 1933 (p. 76), 1942 (p. 92), 1945 (p. 94). Les deux premières sont mentionnées dans le texte de Celan, la demière est absente. 1945, année où la guerre prend fin. Concrètement, historiquement, mais le fantasme ignore le réel et l'histoire.

L'acte n'est pas manqué qui laisse la blessure ouverte, la traduction se tait. Que la guerre ne soit pas finie, la traduction le dit explicitement par un non-dit. Évoquant la vie du commandant du camp, reproduisant la normalité d'une quelconque garnison, dans sa villa et auprès de son épouse, Cayrol remarque : "Peut-être seulement s'y ennuiet-elle un peu plus : la guerre ne veut pas finir " (p. 90). Celan refuse ce demier propos : « [nur] daß die Zeit hier langsamer vergeht... "[le temps seulement passe ici plus lentement...] (p. 91). La fin n'est pas venue et le terme qui clôt le texte de Cayrol est omis, au profit d'une combinaison énonciative différente : $\alpha$ [... nous] qui n'entendons pas qu'on crie sans fin " (p. 98); " [...] und nicht hören, daß der Schrei nicht verstummt * [et qui n'entendons pas que le cri ne se tait pas] (p. 99). Le cri, comme la guerre, ne prend pas fin, il se prolonge dans la traduction qui en recueille l'écho, qui, elle, l'entend et nous le fait entendre.

Et c'est à travers ce cri, cette douleur, le traumatisme répêté grâce à la traduction, que l'expérience de l'horreur est revécue, même pour ceux qui ne l'ont pas connue.

Le Struthof, Oranienburg, Auschwitz, Neuengamme, Belsen, Ravensbruck, Dachau, furent des noms comme les autres sur les cartes 
et les guides. (p. 76)

Struthof, Oranienburg, Auschwitz, Ravensbrïck, Dachau, Neuengamme, Bergen-Belsen :/das waren einmal Namen wie andre, Namen auf Landkarten und in Reisefihrern [ce furent autrefois des noms comme des autres, des noms sur les cartes et dans les guides de voyage]. (p. 77)

Les noms ne sont pas innocents, leur mention est répétée de même que la mention du passé. L'histoire les investit d'un nouvel affect, mais ce nouvel ordre des noms, nouvelle écriture de l'espace, est si insupportable que la traduction les déplace ${ }^{49}$. Isolés et mis en valeur en une ligne séparée, ils ne sont pas donnés dans la même succession. Et la ligne suivante fait apparaître dans le terme allemand pour * guide de voyage " le signifiant Führer connotant cette histoire. Une histoire inédite investissant une géographie inédite, refaite, fantasmatique, que la suppression d'une répétition souligne :

Premier regard sur le camp : c'est une autre planète. Sous son prétexte hygiénique, la nudité, du premier coup, livre au camp l'homme déjà humilie. (p. 80)

Ein erster Blick auf das Lager :/ein anderer Planet./Unter dem Vorwand der Hygiene liefert die Nachtkeit einen bereits Entwürdigten ein. [Premier regard sur le camp :/une autre planète./Sous le prétexte de l'hygiène la nudité livre un (homme) déjà avili.] (p. 81)

Plus bas, d'autres noms topographiques se prêtent à la manipulation traductive. Cayrol cite parmi les lieux des opérations de rafle, d'intemement et de déportation : Varsovie, Lodz, Prague, Bruxelles, Athènes, Zagreb, Odessa, Rome, Pithiviers, le Vel-d'Hiv, Compiègne (p.78). Celan en donne une liste différente : Warschau,

\footnotetext{
${ }^{49} \mathrm{Je}$ vois un processus similaire dans une particularité éditoriale de Rue Ordener, rue Labat de Sarah Kofman (Paris, Galilee, 1994). Dans ce livre qui, comme l'indique le titre, parle de lieux, les titres de chapitres qui forment la table des matières n'apparaissent pas à l'en-tête des sections dans le corps de l'ouvrage, indiquées par les seuls chiffres romains, si bien que les toponymes y figurant (chapitres III, XI, XV, XXIII) disparaissent alors que le texte les mentionne. Les lieux ainsi revisités sont cependant marqués d'un signe d'absence, incomplétude révélant l'affect dont ils sont investis. Le rapport à l'espace est crucial chez nombre d'auteurs écrivant à l'ombre de la Shoah, Georges Perec (voir note suivante), par exemple.
} 
Lodsch, Prag, Brilssel, Wien, Athen, Budapest, Rom, la province française, Paris [" Razzia in der französischen Provinz,/Großfahndung in Paris »] (p. 79). Zagreb et Odessa ('Europe orientale), Pithiviers, le Veld'Hiv, Compiègne disparaissent, mais apparaissent Vienne, Budapest et Paris. Budapest ou, par contiguïté paronomastique, Bucarest : le lieu de la scène de disparition première; Vienne que Celan gagna en quittant Bucarest en 1947, mais qui ne lui offrit pas l'asile rêvé et dont il repartit en 1948 pour Paris. Paris : lieu de la disparition finale, le suicide en 1970 dans les eaux de la Seine. Aux lieux de la disparition historique, Celan mêle, surgis du réel, ceux de sa propre histoire, de sa propre disparition. Traduction : description d'une disparition ${ }^{50}$. Au sens strict chez Celan, mais l'énoncé conviendrait aussi bien à une définition générale. Ce qui a disparu le devient par l'énoncé l'enregistrant, recueillant l'impression en négatif et n'existe, en tant que tel, que la. Logique de l'après coup freudien. Dans $L a$ disparition de Perec, la lettre disparue existe in

5I" * Description d'un chemin *, tel est le titre que donne Perec au chapitre le plus personnel de ses Récits d'Ellis Island (Ed. du Sorbier, 1980). Réminiscence évidente du $\propto$ Description d'un combat $*$ de Kafka, présent dans le livre par une citation des premières lignes de L'Amérique (p. 48). Or ce chapitre commence et se conclut par la mention des pays quittés par les émigrants/immigrants (pp. 19 et 50), c'est-à-dire des lieux de leur disparition. Au demeurant, Perec confie : * [...] ce que moi, Georges Perec, je suis venu questionner ici, c'est l'errance, la dispersion, la diaspora./Ellis Island est pour moi le lieu mêrne de l'exil, c'est-à-dire le lieu de l'absence de lieu, le non-lieu, le nulle-part " (p. 42). Ce dernier terme est fréquent et majeur dans la poésie celanienne. Par exemple : " L'ÉCRIT SE CREUSE, le/dit, vert-océan,/brûle dans les baies, /dans les/noms liquéfiés/bondissent les marsouins,/dans le nulle-part rendu éternel, icimême,/dans la mémoire des/cloches toutes bruyantes dans - où donc? [...] * (Strette et autres poèmes, tr. Jean Daive, Paris, Mercure de France, 1990, p. 93). Le vide permet l'écriture, non pour qu'il soit comblé mais comme seul moyen de le dire, thème familier de la littérature analytique. L'obsession des lieux chez Perec naît de la même motivation que chez Celan, d'un deuil impossible : un lieu a disparu, celui de la disparition (de la mère, notamment) et l'écriture le recueille. " Au plus fort du Logos, il y a un champ proscrit, tabou zonal dont aucun n'approchait, qu'aucun soupcon n'indiquait : un Trou, un Blanc, signal omis qui, jour sur jour, prohibait tout discours, laissait tout mot vain, brouillait la diction, abolissait la voix dans la maldiction d'un gargouillis strangulant * (La disparition, Paris, Denoël, 1979, p. 129). Cette dernière proposition résonne étrangement avec " l'occlusion laryngale * citée au début du présent texte. Rappeler alors, pour prolonger le fantasme, que Perec mourut d'un cancer des bronches. 
absentia dans le livre qui n'existe que par elle, autour d'elle, de son manque. De même, l'original disparaît dès lors qu'il est traduit et existe disparu par et dans la traduction. L'original devient originel, un Urtext.

Les noms qui disparaissent sans exception sont, dans le passage qui précède celui tout juste cité, les noms propres de personnes, dans l'implacable logique du réel que le transfert traductif permet d'accepter.

Pendant ce temps, Burger, ouvrier allemand, Stern, étudiant juif d'Amsterdam, Schmulzki, marchand de Cracovie, Annette, lycéenne de Bordeaux, vivent leur vie de tous les jours, sans savoir qu'ils ont déjà, à mille kilomètres de chez eux, une place assignée./Et le jour vient où leurs blocks sont terminés, où il ne manque plus qu'eux. (p. 78) Inzwichen geht das Leben seinen Gang; der Arbeiter aus Berlin, der jüdische Student aus Amsterdam, der Kaufman aus Krakau, die Lyzealschülerin aus Bordeaux : sie alle ahnen nicht, daß ihnen in einer Entfernung von Tausend Kilometernn bereits ein Platz zugewiesen ist. Und dann kommt der Tag, an dem ihre Blocks fertig sind/und nur sie noch fehlen. [Pendant ce temps la vie suit son cours; l'ouvrier de Berlin, l'étudiant juif d'Amsterdam, le marchand de Cracovie, la lycéenne de Bordeaux : aucun d'entre eux ne se doute que, à une distance de mille kilomètres, une place leur est déjà assignée. Et puis vient le jour où leurs blocks sont terminés/et il ne manque plus qu'eux.] (p. 79)

La disparition des (noms de) personnes est d'autant plus flagrante qu'un nom de ville est ajoutée (Berlin). Thêâtralité spectrale, la mise en mots est mise en morts. Si définitive la mise à mort qu'elle en supprime la vie antérieure. Si profond le traumatisme que la traduction celanienne fantasme un monde à jamais, c'est-à-dire depuis toujours dévitalisé, peuplé de fantômes, alors que la poésie celanienne accueille dans le dialogue les disparus, leur redonnant vie ${ }^{52}$. Plus de noms de personnes, que des noms de lieux : des scènes vides où le vide est mis en scène. De même que plus bas, là où Cayrol emploie " raflés v et a déportés ", Celan dit "Auhebungen " et * Aussiedlungen" désignant les opérations ellesmêmes et non leurs victimes. Que des morts : " Aujourd'hui sur la même voie, il fait jour et soleil. On la parcourt lentement, à la recherche de quoi? De la trace des cadavres qui s'écroulaient dès l'ouverture des portes? " (pp.

\footnotetext{
sI Par exemple, les récurrences de l'adresse à la deuxième personne ou de la figure de la sour.
} 
78-80); " Dieselbe Bahnstrecke heute : Tageslicht und Sonne. Langsam schreitet man sie ab - auf der Suche wonach?/Nach einer Spur der Leichen? " [La même voie ferrée aujourd'hui : lumière du jour et soleil. On la parcourt lentement - à la recherche de quoi?/D'une trace des cadavres?] (pp. 79-81). Le détail narratif importe peu. Que des cadavres existant en tant que tels, quelles que soient les circonstances ayant entraîné la mort (ici le transport dans les wagons).

La désignation même des déportés offre des variations qui sont autant de déplacements d'affects. En deux occurrences dans le texte, " déportés " est traduit par le terme correspondant * Verschleppte " (pp. 78-79) ou " le déporté " par le pluriel " die Deportieren " (pp. 80-81). Dans la plupart des cas (pp. 84-85, 86-87, 90-91, 92-93), “ déporté *, au singulier ou au pluriel, est traduit par $~ K Z$-Höfling " ou $~ H$ Haftling " (pl. : "KZ-Häflinge ", "Häflinge "), qui est le terme administratif (littéralement : le détenu du camp de concentration), le plus proche donc de la réalité que le nazisme dissimulait sous une froideur comptable, mais dont cette dissimulation accentue encore l'horreur. Le choix lexical de la traduction opère ainsi comme un dévoilement de la dénégation ${ }^{52}$. Le camp doit se dire dans la langue du camp. Inexorablement : là où Cayrol oublie le terme précis, disant * chefs * (p. 96) alors qu'auparavant il employait " prominents " (p. 90) pour désigner $\alpha$ le gratin du camp $*$, Celan répète « Prominente (p. 97).

En deux endroits, cependant, un autre mode de représentation commande la traduction : * [ les soldats] visaient sans fin les déportés, les tuaient à l'occasion, par déscuuvement \# (p. 86); « [...] und gelegentlich

\footnotetext{
${ }^{2} \alpha$ Hăftling : j'ai appris que je suis un Hăftling. Mon nom est 174517 ; nous avons été baptisés et aussi longtemps que nous vivrons nous porterons cette marque tatouée sur le bras gauche * (Si c'est un homme [tr. Martine Schruoffeneger], Paris, Julliard, 1987, p. 31). Primo Levi adopte cette même auto-désignation et son livre explore tous les aspects de cette identité qui touche une dimension sociologique autant qu'ontologique. C'est la micro-société du camp qu'il décrit en même temps qu'une condition humaine inédite : $\propto$ En un instant, dans une intuition quasi prophétique, la réalité nous apparaît : nous avons touché le fond. Il est impossible d'aller plus bas : il n'existe pas, il n'est pas possible de concevoir condition humaine plus misérable que la nôtre ^ (ibid., p. 30). Voir aussi Robert Antelme, L'Espèce humaine.
} 
den einen oder andern abschießen, aus Langweile * [et a l'occasion en abattaient un ou un autre, par ennui] (p. 87). Les victimes n'ont plus de désignation, n'en ont plus besoin, déjà morts de leur seule présence au camp. Statut d'absence, identité anéantie ou plutôt identité de néant, que

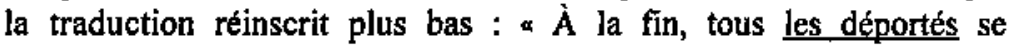
ressemblent. Ils s'alignent sur un modèle sans âge qui meurt les yeux ouverts" (p. 88); "Zuletzt haben alle das gleiche Gesicht. Es sind alterslose Wesen, die mit offenen Augen sterben [ $[\grave{A}$ la fin tous ont le même visage. Ce sont des êtres sans âge qui meurent les yeux ouverts] ( $p$. 89). La logique de la disparition est menée à son terme : les victimes abandonnent même le statut qui les fait disparaître, acquérant une dimension que je qualifierai de thanato-ontologique, que la traduction souligne par le lexique philosophique de l'être. Ce qu'a accueilli l'histoire ne peut être traduit que par un déplacement vers le non-historique.

En revanche, à la dernière mention dans le texte, la substitution opérée se motive d'un affect positif. Cayrol évoque la libération des camps : "Les déportés regardent sans comprendre. Sont-ils délivrés?"; " sehen die Öberlebenden zu, ohne zu begreifen. Sind sie befreit?" (pp. 96-97). Le terme utilisé par Celan est celui qui désigne les rescapés, les survivants. Pour eux la nuit a pris fin. Et à cet endroit, Celan l'inscrit dans sa traduction. Quelques lignes plus loin, cet apaisement lui fera modifier, l'atténuant, le texte de Cayrol, en en omettant une proposition : La guerre s'est assoupie, un ceil toujours ouvert " (p. 96); * Der Krieg schlummert nur w (p. 97). Non que ce passage soit sauf de l'ambivalence fantasmatique : dans la phrase précédente, là où Cayrol dit * charniers ", Celan dit \& Totenkammern ", " chambres de mort " (doublet de Gazkammern, chambres à gaz). Au terme trop général est substitué celui dicté par le principe de réalité et le devoir d'historicité.

La blessure mélancolique, un instant refermée, se rouvre. * L'herbe fidèle est venue à nouveau sur les appel-platzs autour des blocks " (p. 96); * [...] hat sich wieder das Gras angesiedelt " [... l'herbe s'est réinstallée] (p. 97). L'herbe n'est pas fidèle, elle n'est qu'elle-même, la nature dans sa cruelle naturalité, sans adjectivation d'un lexique moral, c'est-à-dire humain, car l'humain n'a plus sa place dans ce lieu, depuis qu'il en a été définitivement expulsé, depuis que cette élimination en a signé la spécificité. 
Dans les dernières lignes du texte, Celan traduit fidèlement le pessimisme de Cayrol, le soulignant même d'une répétition : "Neuf millions de morts hantent ce paysage * (p. 96); "Diese Landschaft : die Landschaft von neuen Millionen Toten " [Ce paysage : un paysage de neuf millions de morts] (p. 97). Comme au début du texte, l'insistance est sur le lieu, la scène (du) vide, la scène finale devenue originaire, traumatique.

Une scène vide, un texte où disparaissent les noms des disparus. Les noms ont cependant été prononcés, dans l'original, $\mathrm{C}^{\prime}$ est donc la traduction qui rejoue leur disparition. Mais ce faisant, elle transforme leur statut. Ils deviennent dissimulés, cachés, existants mais secrets, sécrétés par la traduction au secret du texte premier. Là où chez Proust, par exemple, le nom propre - Gilberte, Guermantes, ... - traverse l'histoire et la contient, chez Celan, il lui est soustrait. L'historicité différente des deux écritures commande ces traitements distincts.

D'une manière générale, la traduction est cryptage en même temps que décryptage puisqu'elle rend, en le dédoublant, secret, caché, absent, ce qui ne l'était pas dans l'intégrité de l'énoncé premier devenu, dès lors, second. Elle contient cryptiquement l'original, dans son for intérieur et elle est seule à en (s)avoir le secret. C'est elle qui crée l'original. Dans la cohésion interne du système freudien, le paradigme traductif est un autre phénomène de Nachträglichkeit. Après coup qui permet à Celan de dire la Shoah en traduisant le texte de Cayrol comme un avant-dit ${ }^{53}$.

Le nom de Celan lui-même, pseudonyme, est le masque du nom patronymique, Antsche $\left.\right|^{\text {s4 }}$. Simple renversement des syllabes, le patronyme est bien conservé cryptiquement. Et Celan, francophone, ne

${ }^{51}$ Jean-Michel Rey a montré comment pour Freud aussi l'histoire ne pouvait s'écrire que comme après coup (* Freud et l'écriture de l'histoire ", $L ' E$ '́crit $d u$ temps, $\left.\mathrm{n}^{\circ} 6,1984\right)$.

${ }^{54}$ Voir n. 13. 
pouvait ignorer l'homonymie : Celan/celant/scellant ${ }^{55}$. La dissimulation se pare de l'incertitude des données biographiques quant à la date, le choix ou l'attribution de ce pseudonyme : le don du secret demeure secret, l'ouverture de la crypte reste cryptée.

Les noms ne disparaissent donc pas tant qu'ils sont cachés, protégés ${ }^{56}$. La traduction celanienne fantasme leur survie, leur sauvegarde. De la dimension fantasmatique du rêve et du symptôme, Maria Torok et Nicolas Abraham écrivent : " Tous deux, par les paroles qui les énoncent, renvoient à des mots qui ne s'énoncent pas et qui se localisent, en tant que tels, à l'intérieur de la crypte ${ }^{57}$. Car la crypte est un lieu. Le nom peut disparaître si un lieu le protège, qui, lui, ne peut disparaître ${ }^{58}$. Les toponymes demeurent dans la traduction celanienne ${ }^{59}$. Une scène vide est toujours une scène. Le nom d'une ville n'est propre à personne, mais appartient à une multiplicité de sujets qu'il désigne. Lorsque Celan le fait disparaître, comme on l'a va plus haut, c'est métonymiquement sa propre disparition qu'il inscrit.

Ce qui amène à poser une hypothèse quant à la traduction des noms propres (ou à leur manipulation dans les mécanismes inconscients) qui ne seraient pas tous de la même manière traduisibles. En maintenant

\footnotetext{
55 * Scelle " , dernier mot fermant la conclusion, portant sur la valeur des noms, du texte de Derrida introduisant Cryptonymie. Le verbier de l'Homme aux loups de Nicolas Abraham et Marja Torok (Paris, Aubier Flammarion, 1976, p. 73). Schibboleth s'intitule, significativement, le livre que Derrida consacra à Celan (voir n. 8). D'autres scellés se posèrent à notre regard, au départ du présent texte.

${ }^{56}$ Sur le secret en tant que sauvegarde des noms, voir mon article * Noms secrets et noms sacrés ", dans Le texte et le nom (sous la direction de Martine Léonard et Élisabeth Nardout-Lafarge), Montréal, XYZ éditeur, 1996.
}

${ }^{57}$ Op. cit., p. 235.

5B Voir dans l'introduction de Jacques Derrida à l'ouvrage pré-cité le développement sur la nature spécifique de la spatialité inhérente à la crypte. Il la relie, fort pertinemment pour notre propos, a l'atopocité de la mort.

${ }^{54}$ Ils occupent au demeurant une position crtuciale dans les poèmes, ce dont l'étude outrepasserait le cadre du présent article. 
les toponymes et supprimant les anthroponymes, la traduction de Celan suggèrerait qu'un nom de lieu appartient a la langue quand un nom de personne lui échappe ${ }^{60}$. Auschwitz est resté Auschwitz, c'est-à-dire saisi dans la langue allemande. Le toponyme ne peut être enfermé dans la crypte car il est crypte, ou sépulture. À ce titre, il peut supporter, susciter un travail de deuil impossible pour l'anthroponyme. Et le texte y déposera die Niemandsrose, la rose de personne ${ }^{6 i}$.

Cela, deux poèmes le disent, dans Zeigehoft, le demier des trois recueils posthumes, celui qui scelle l'ceuvre poétique : « DAS NICHTS, um unser/Namen willen-sie sammeln uns ein -/siegelt [...]"; " LE NÉANT, au nom/de nos noms $/$ - ils nous recueillent - ,/appose les scellés [...] ${ }^{62}$. Première personne du pluriel, le nous identificatoire et fusionnel, crée dans le processus mélancolique où a l'ombre de l'objet tomba sur le moi ${ }^{63}$, les deux pertes se confondant.

L'objet perdu retrouvé, il est alors possible de traduire les mots du deuil :

[...] der Chor/der Platanenstrünke/buckelt sich ein zum Gebet/gegens Gebet,//aus gesiegeltem Floßholz/bau ich dir Namen, die pflockst duffest, bei den Regenfeimen [...]

\footnotetext{
${ }^{60}$ On reconnaîtra ici la problématique introduite par Jacques Derrida dans * Des tours de Babel *, Psyché. Inventions de l'autre, Paris, Galilée, 1987. Voir aussi L'Oreille de l'autre (sous la direction de Claude Lévesque et Christie McDonald), Montréal, VLB éditeur, 1982.

6. « Un rien/nous étions, nous sommes, nous/resterons, en fleur :/a rose de rien, de/personne * ( Psaume , dans La rose de personne, tr. M. Broda, Paris, Le Nouveau Commerce, 1979, p. 39).

${ }^{62}$ Enclos du temps, tr. Martine Broda, Paris, Clivages, 1985, p. 91.

${ }^{63}$ « Deuil et mélancolie ", p. 156.
} 
[...] le chour/des souches de platane/se met en boule pour la prière/contre la prière,//en bois flotté scellé/je bâtis des noms pour toi, qui les fixes/avec des chevilles, auprès des meules de la pluie $[\ldots]^{64}$

\section{Université de Montréal}

\section{Références}

ABRAHAM, Nicolas et Maria TOROK (1976). Cryptonymie. Le verbier de l'Homme aux loups. Paris, Aubier-Flammarion.

ANTELME, Robert (1978). L'espèce humaine. Paris, Gallimard, « TEL". ANZIEU, Didier (1981). Le Corps de l'auvre. Paris, Gallimard.

BERMAN, Antoine (1984). L'éprewve de l'étranger. Paris, Gallimard. BRODA, Martine (1986). Dans la main de personne. Essai sur Paul Celan. Paris, Cerf.

CAYROL, Jean (1997). Nuit et brouillard. Paris, Fayard, " Libres n.

CELAN, Paul (1993). Grille de parole. Tr. M. Broda. Paris, Christian Bourgois éditeur.

-(1991). De seuil en seuil. Tr. V. Briet. Paris, Christian Bourgois éditeur.

- (1990). Strette et autres poèmes. Tr. J. Daive. Paris, Mercure de France.

- (1990). Entretien dans la montagne. Tr. S. Mosès. Paris, Ed. Michel Chandeigne.

- (1989). Contrainte de lumière. Tr. B. Badiou et J.C. Rambach. Paris, Belin.

- (1987). Pavot et mémoire. Tr. V. Briet. Paris, Christian Bourgois éditeur.

${ }^{64}$ Enclos du temps, pp. 14-15. 
- (1987). Poèmes. Tr. J.E. Jackson. Le Muy, Éditions Unes.

- (1985). Enclos du temps. Tr. M. Broda. Paris, Clivages,

- (1983). Atemwende, Fadensonnen. Dans Gesammelte Werke in fünf Bänden. Frankfurt am Main, Surtkamp.

- (1979). ^Le Méridien ‥ Tr. J. Launay. Podsie, n 9.

- (1979). La rose de personne. Tr. M. Broda. Paris, Le Nouveau Commerce.

DERRIDA, Jacques (1987). Psyché. Inventions de l'autre. Paris, Galilée.

- (1986). Schibboleth. Pour Paul Celan. Paris, Galilée.

- (1982). L'Oreille de l'autre. Montréal, VLB éditeur.

FELSTINER, John (1995). Paul Celan : Poet, Survivor, Jew. New Haven and London, Yale University Press.

FREUD, Sigmund (1986). Métapsychologie. Paris, Folio.

GADAMER, Hans-Georg (1987). Qui suis-je et qui es-tu ? Commentaire de Cristaux de souffle de Paul Celan. Arles, Actes Sud.

HASSOUN, Jacques (1995). La cruauté mélancolique. Paris, Aubier.

KAES, René (1994). La parole et le lien. Paris, Dunod.

KOFMAN, Sarah (1994). Rue Ordener, rue Labat. Paris, Galilee.

-(1975). L'Enfance de l'art. Paris, Payot.

KRISTEVA, Julia (1987). Soleil noir. Dépression et mélancolie. Paris, Gallimard.

LEVI, Primo (1987). Si c'est un homme. Paris, Julliard. 
NOUSS, Alexis (1998), * Mémoire et survie : une lecture de Paul Celan *. Études françaises, $34: 1$, printemps.

- (1997). * L'abandon du traducteur * (tr. Laurent Lamy et Alexis Nouss). TTR, vol. $\mathrm{X}, \mathrm{n}^{\circ} 2$.

- (1996). « Dans la ruine de Babel : poésie et traduction chez Paul Celan *. TTR, vol. IX, $\mathrm{n}^{\circ} \mathrm{I}$.

PERALDI, François (1982). * Psychanalyse et traduction *. Dans META, $27: 1$.

- (1979). La disparition. Paris, Denoël.

PEREC, Georges (1980). Récits d'Ellis Island. Paris, Éditions du Sorbier.

REY, Jean-Michel (1984). " Freud et l'écriture de l'histoire ". Dans L'Écrit du temps, $\mathrm{n}^{\circ} 6$.

- (1979). Des mots à l'auvre. Paris, Aubier-Montaigne.

STENER, George (1998). Errata. Récit d'une pensée. Paris, Gallimard.

- 1991). Réelles présences. Les arts du sens. Paris, Gallimard.

- (1987). Passions impunies. Paris, Gallimard.

- (1978). Après Babel. Une poétique du dire et de la traduction. Paris, Albin Michel.

WILKOMIRSKI, Benjamin (1997). Fragments. Une enfance. Paris, Calman-Lévy.

RÉSUMÉ : La traduction mélancolique (sur Paul Celan) - Dans l'œuvre de Paul Celan, poèmes et traductions (en majorité des poèmes, du $\mathrm{XVI}^{\bullet}$ au $\mathrm{XX}^{*}$ siècles, à partir de sept langues) participent d'une même poétique dont la fonction est de retrouver une langue atteinte par la ténèbre du nazisme et d'opérer un travail de deuil. La stratégie scripturaire des traductions, cependant, est spécifique en ce qu'elle permet un dire 
affrontant le trauma. À l'aide de notions freudiennes, notamment le transfert et la mélancolie, nous étudions ce processus à travers la traduction du commentaire de Jean Cayrol écrit pour le film Nuit et brouillard.

\begin{abstract}
Melancholic Translation (on Paul Celan) - In Celan's work, poems and translations (mostly poems, from the $\mathrm{XVI}^{\text {th }}$ to the $\mathrm{XX}^{\text {th }}$ centuries and from seven languages) belong to a same poetics whose function is to restore a language affected by the darkness of nazism and to carry out a work of mourning. The writing strategy of the translations, nonetheless, is specific since it allows a * saying " confronting the trauma. With the help of Freudian notions, especially transference and melancholia, we study this process through the translation of the commentary written by Jean Cayrol for the film Night and Fog.
\end{abstract}

Alexis Nouss : Département de linguistique et de traduction, Université de Montréal, C.P. 6128, succ. Centre-ville, Montréal (Québec) H3C 3J7. 